\title{
Clipping versus Coiling in Ruptured Aneurysms of the Anterior Cerebral Circulation
}

\author{
Farrag Mohammad1,2* (D), Takashi Horiguchi'1, Katsuhiro Mizutani1, Kazunari Yoshida1 \\ ${ }^{1}$ Department of Neurosurgery, Keio University School of Medicine, Tokyo, Japan \\ ${ }^{2}$ Department of Neurosurgery, Faculty of Medicine, Assiut University, Assiut, Egypt \\ Email: ^farragmohammad@aun.edu.eg, ^farragmohamad@gmail.com
}

How to cite this paper: Mohammad, F., Horiguchi, T., Mizutani, K. and Yoshida, K. (2020) Clipping versus Coiling in Ruptured Aneurysms of the Anterior Cerebral Circulation. Open Journal of Modern Neurosurgery, $10,88-104$.

https://doi.org/10.4236/ojmn.2020.101010

Received: November 19, 2019

Accepted: December 10, 2019

Published: December 13, 2019

Copyright () 2020 by author(s) and Scientific Research Publishing Inc. This work is licensed under the Creative Commons Attribution International License (CC BY 4.0).

http://creativecommons.org/licenses/by/4.0/

\begin{abstract}
Objective: To evaluate and compare the outcomes of microsurgical clipping and endovascular coiling of ruptured anterior cerebral circulation aneurysms. Methods: The current study retrospectively included 61 patients presented with subarachnoid hemorrhage (SAH), treated by clipping or coiling for ruptured aneurysms located in anterior circulation. Anatomic outcome and post-operative ischemic events were evaluated. The primary clinical outcomes were evaluated as operative complications while the secondary clinical outcome was evaluated by modified Rankin scale (mRS) at discharge. Results: Of 61 ruptured aneurysms, 47 and 14 were treated by surgery and coiling, respectively. The average follow-up duration was $19.1 \pm 18.6$ months for clipping and $21.7 \pm 14.8$ months for coiling. Complete occlusion was greater in surgery group (80.8\%) than endovascular group (21.4\%). Re-growth occurred in 2 cases of the coiling group only. Ischemic events were encountered in both groups; silent ones were higher in coiling group (21.4\%) than in clipping group $(6.3 \%)$, while symptomatic ones were higher in the clipping group (49\%). Good outcome (mRS 0 - 2) was $51 \%$ and $71.4 \%$ after clipping and coiling respectively. Postoperative hospital period was longer after clipping $(\mathrm{P}$ $=0.04)$. Conclusions: For anterior circulation ruptured aneurysms, both treatments are feasible, coiling showed lower rate of morbidity while clipping results were better regarding durability and recurrence. Coil embolization may be preferred, which is the current point proved by the RCT like ISAT, but if you choose the patients carefully and find out the optimal candidate for the clipping, the clipping is still enough feasible as a mainstream.
\end{abstract}

\section{Keywords}

Anterior Circulation, SAH, Clipping, Coiling, Ruptured Aneurysms 


\section{Introduction}

Ruptured cerebral aneurysms are considered the leading cause of non-traumatic subarachnoid hemorrhage (SAH) and represent $70 \%-80 \%$ of all SAH cases [1]. Untreated ruptured intracranial aneurysms cause a high burden of morbidity and mortality with a high risk of rebleeding [2]. Treatment of ruptured aneurysms is difficult and different from treatment of unruptured ones. The difficulty comes from factors caused by the aneurysm rupture and $\mathrm{SAH}$ as brain swelling, presence of hematoma, the resulting intracranial hypertension, vasospasm, and fragility of the aneurysm at the site of rupture with increased risk of intraprocedural rupture [3].

Endovascular treatment (EVT) of intracranial aneurysms was initially introduced as a means of occluding inoperable aneurysms. Since the development of electrolytically detachable coils, the indications for EVT have widened to include surgically difficult ruptured, as well as non-ruptured, aneurysms [4]. Since then several advancements, including complex-shaped coils, balloon remodeling techniques, the use of stent-supported coiling, or flow diverter stents, have been introduced into the endovascular treatment options for more anatomically difficult and complex aneurysms leading to increased use of endovascular treatment in the management of cerebral aneurysms [5].

Endovascular coil embolization and surgical clipping are the main treatment options for ruptured and unruptured aneurysms. Which treatment modality is best in terms of aneurysm occlusion and durability with low recurrence rate and post-operative morbidity is, however, a widely debated topic. In this study we assess both modalities in the treatment of ruptured aneurysms in the anterior cerebral circulation.

\section{Materials and Methods}

The current study retrospectively included patients presented to Keio hospital by subarachnoid hemorrhage due to ruptured Saccular aneurysms located in anterior cerebral circulation from January 2012 to December 2017 with exclusion of patients with giant $(>25 \mathrm{~mm})$, fusiform or dissecting aneurysms, patients unfit for surgery or endovascular management were also excluded. All patients were initially admitted to intensive care unit. The management of SAH patients was performed according to the AHA/ASA latest guidelines [6]. All patients requiring external ventricular drainage were so treated, and all patients received appropriate vasospasm prophylaxis and treatment in the form of nimodipine 60 mg orally every 4 hours and maintenance of euvolemia.

The indication of surgical or interventional treatment was decided by evidence-based manner. The selection of treatment for each case was based on characteristics of individual patients and aneurysms offering both modalities of treatment taking into account patient preference. All clipping and coiling cases were performed on with monitoring of motor evoked potential (MEP) and somatosensory evoked potential (SEP). In clipping cases, the patency of parent artery, major branches, and visible perforators were confirmed with laser Doppler 
flowmetry and indocyanine green angiography.

\subsection{Angiographic Outcome}

Anatomic results were divided into complete occlusion, presence of postoperative neck remnant and re-growth on follow-up. Postoperative CT images were obtained in the first and seventh postoperative days after clipping. In coil embolization, CT was obtained in the first postoperative day and MRI in the fifth postoperative day, other follow up imaging was done at variable intervals depending on the case. Imaging included CT angiography (CTA), MRA and/or digital subtraction angiography (DSA).

\subsection{Ischemic Events and Clinical Outcomes}

The primary outcomes were evaluated as operative complications such as ischemic events, neurological morbidity (irrespective of duration), the presence of post-procedure hemorrhage (subdural, extradural, cerebral and subarachnoid hemorrhage), or infection (wound infection, meningitis, and pneumonia).

Ischemic events were defined by the presence of infarction on subsequent CT scan or MRI or angiographic occlusion of a blood vessel by the time of hospital discharge regardless of the presence of symptoms. In the current study early infarction (post-procedure in the first 3 days of SAH) is considered procedure-related complication while late infarction (4 days after the start of SAH) is considered due to vasospasm, some cases exhibited both types.

The secondary clinical outcome was evaluated by worsening of modified Rankin scale (mRS) at discharge, mRS was used for evaluation considering mRS 0 - 2 as good outcome and mRS 3 - 6 as poor outcome.

\subsection{Independent Variables}

Independent variables investigated as possible risk factors for outcomes included demographic data of patients (age, sex, preoperative WFNS grade, preoperative SAH grade, presence of diabetes mellitus, hyperlipidemia, hypertension and other co-morbidities), aneurysm data (dome size, aneurysm height, neck width, presence of daughter sac, incorporate artery and multiple aneurysms), procedure-related data (temporary arterial occlusion in surgery-the use of balloon and stent in coiling) and follow up duration.

\subsection{Statistics}

Categorical variables expressed as a percentile, continuous variables as mean \pm SD. Univariate analysis is done using chi-square test, Fisher exact test, and unpaired t-test as appropriate. Multivariate analysis is conducted by multivariate logistic regression.

\subsection{Informed Consent}

Informed consent is obtained from the patients by the way of the opt-out condi- 
tion. The present study was approved by the ethics committee of Keio University School of Medicine and was performed in accordance with the ethical standards of the 1964 Declaration of Helsinki and its later amendments.

\section{Results}

\subsection{Characteristics of Patients and Aneurysms}

Table 1 shows baseline characteristics of patients and aneurysms. Of 61 ruptured

Table 1. Demographic criteria of patients and aneurysms for ruptured cases.

\begin{tabular}{|c|c|c|c|}
\hline Characteristics & Microsurgery (47) & Endovascular (14) & $P$ value \\
\hline Age (year) & $58.8 \pm 14$ & $56 \pm 16$ & 0.57 \\
\hline \multicolumn{4}{|l|}{ Gender } \\
\hline Female & $28(59.6 \%)$ & $10(71.4 \%)$ & 0.53 \\
\hline Male & $19(40.4 \%)$ & $4(28.6 \%)$ & 0.53 \\
\hline Hypertension & $25(53 \%)$ & $5(35.7 \%)$ & 0.25 \\
\hline $\mathrm{DM}$ & $3(6.4 \%)$ & $1(7.1 \%)$ & 0.91 \\
\hline Hyperlipidemia & $4(8.5 \%)$ & 0 & 0.56 \\
\hline Other co morbidities & $22(46.8 \%)$ & $6(42.8 \%)$ & 0.79 \\
\hline \multicolumn{4}{|l|}{ WFNS grade } \\
\hline Grade 1 & $13(27.6 \%)$ & $4(28.6 \%)$ & 0.94 \\
\hline Grade 2 & $14(29.8 \%)$ & $3(21.4 \%)$ & 0.7 \\
\hline Grade 3 & $2(4.2 \%)$ & 0 & 0.99 \\
\hline Grade 4 & $12(25.6 \%)$ & $3(21.4 \%)$ & 0.75 \\
\hline Grade 5 & $6(12.8 \%)$ & $4(28.6 \%)$ & 0.2 \\
\hline \multicolumn{4}{|l|}{ Modified Fisher grade } \\
\hline Grade 1 & $3(6.4 \%)$ & $2(14.3 \%)$ & 0.3 \\
\hline Grade 2 & $3(6.4 \%)$ & $2(14.3 \%)$ & 0.3 \\
\hline Grade 3 & $29(61.7 \%)$ & $6(42.8 \%)$ & 0.2 \\
\hline Grade 4 & $12(25.5 \%)$ & $4(28.6 \%)$ & 0.8 \\
\hline \multicolumn{4}{|l|}{ Aneurysm criteria } \\
\hline \multicolumn{4}{|l|}{ Location of aneurysm } \\
\hline ICA & $11(23.4 \%)$ & $8(57 \%)$ & $0.016^{*}$ \\
\hline $\mathrm{MCA}$ & $16(34 \%)$ & $1(7 \%)$ & $0.04^{*}$ \\
\hline ACA & $20(42.6 \%)$ & $5(36 \%)$ & 0.64 \\
\hline Maximum diameter of dome (mm) & $3.9 \pm 2.2$ & $5.4 \pm 3.1$ & 0.11 \\
\hline Maximum height of the sac (mm) & $5 \pm 2.4$ & $5.2 \pm 1.8$ & 0.78 \\
\hline Neck width $(\mathrm{mm})$ & $2.8 \pm 1.4$ & $2.6 \pm 0.8$ & 0.56 \\
\hline Presence of daughter sac & $28(59.6 \%)$ & $11(78.6 \%)$ & 0.22 \\
\hline Presence of Incorporate artery (IA) & $6(12.7 \%)$ & $3(21.4 \%)$ & 0.41 \\
\hline ICA & $6 / 11(54.5 \%)$ & $2 / 8(25 \%)$ & 0.35 \\
\hline $\mathrm{MCA}$ & $0 / 16$ & $1 / 1(100 \%)$ & 0.05 \\
\hline ACA & $0 / 20$ & $0 / 5$ & \\
\hline \multicolumn{4}{|l|}{ Operative: } \\
\hline Temporary arterial occlusion in surgery & $34(72.3 \%)$ & & \\
\hline Balloon-assisted coiling & & $8(57 \%)$ & \\
\hline Stent-assisted coiling & & 0 & \\
\hline
\end{tabular}

DM: Diabetes mellitus; ICA: internal carotid artery; MCA: middle cerebral artery; ACA: anterior cerebral artery; IA: Incorporate artery; WFNS: World Federation of Neurosurgical Societies; ${ }^{*}$ statistically significant. 
aneurysms, 47 and 14 were treated by surgery and coiling, respectively. Between treatment groups, the location of aneurysm showed significant difference. In coiling group, 8 aneurysms (57\%) were located at ICA. In contrast ruptured aneurysms located at MCA treated by coiling were only 1 (7\%). There were no other significant differences between the two groups.

\subsection{Angiographic Outcome}

Complete obliteration of the ruptured aneurysms was greater in clipping group (80.8\%) than in the coiling group $(21.4 \%)(\mathrm{P}=0.0005)$, the frequency of post-operative neck remnant was greater in the coiling group $(71.4 \%)$ than in the clipping group $(19.2 \%)(\mathrm{P}=0.0005)$. Regrowth occurred in 2 cases after coiling (14.2\%) both continued under follow up while no regrowth occurred after clipping $(\mathrm{P}=0.04)$. In one case coiling was renounced and patient did not receive any further treatment (Table 2 ).

\subsection{Clinical Outcomes}

The average follow-up duration for ruptured aneurysms was $19.1 \pm 18.6$ months for the surgery group and $21.7 \pm 14.8$ months for the coiling group. Good outcome obtained in 24 cases in the surgery group (51\%) and 10 cases in the endovascular

Table 2. Summary of results for ruptured cases.

\begin{tabular}{|c|c|c|c|}
\hline Outcome & Microsurgery (47) & Endovascular (14) & $P$ value \\
\hline Complete occlusion & $38(80.8 \%)$ & $3(21.4 \%)$ & $0.0005^{\star}$ \\
\hline Neck remnant & $9(19.2 \%)$ & $10(71.4 \%)$ & $0.0005^{*}$ \\
\hline Re growth & 0 & $2(14.2 \%)$ & $0.04^{*}$ \\
\hline Retreatment for regrowth & 0 & 0 & \\
\hline Failure & 0 & $1(7.2 \%)$ & 0.22 \\
\hline Rebleeding & $1(2.1 \%)$ & $1(7.2 \%)$ & 0.4 \\
\hline Asymptomatic thromboembolism & $3(6.3 \%)$ & $3(21.4 \%)$ & 0.12 \\
\hline Symptomatic thromboembolism & $23(49 \%)$ & $5(35.7 \%)$ & 0.38 \\
\hline $\mathrm{SDH} / \mathrm{EDH}$ & $6+7(27.6 \%)$ & 0 & $0.02^{*}$ \\
\hline Contusions/hemorrhage & $3+2(10.6 \%)$ & 0 & 0.58 \\
\hline Hydrocephalus & $13(27.6 \%)$ & $4(28.6 \%)$ & 0.94 \\
\hline Temporary morbidity & $18(38.3 \%)$ & $3(21.4 \%)$ & 0.34 \\
\hline Permanent morbidity & $16(34 \%)$ & $5(35.7 \%)$ & 0.9 \\
\hline Operations for complications & $29(61.7 \%)$ & $4(28.6 \%)$ & $0.036^{*}$ \\
\hline wound infection/meningitis & $5(10.6 \%)$ & 0 & 0.58 \\
\hline Pneumonia & $16(34 \%)$ & $4(28.6 \%)$ & 0.7 \\
\hline Post-operative hospital period (day) & $44 \pm 25$ & $31.4 \pm 18$ & $0.04^{\star}$ \\
\hline Mortality & $1(2.1 \%)$ & $2(14.2 \%)$ & 0.12 \\
\hline
\end{tabular}

SDH: subdural hematoma; EDH: extradural hematoma; ${ }^{*}$ statistically significant. 
group $(71.4 \%)$ with no significant statistical difference $(\mathrm{P}=0.22)$. Nonetheless, as shown in Table 3, the outcome of endovascular treatment in severe cases (WFNS 4 - 5) was much better than surgery as good outcome was obtained in $57.1 \%$ of cases with coiling and $16.7 \%$ of cases with clipping but was not statistically significant $(\mathrm{P}=0.06)$. Regarding mortality, one case $(2.1 \%)$ in the surgery group and two cases in the endovascular group died $(14.2 \%)(\mathrm{P}=0.12)$, all of them were WFNS grade 5 (Table 4).

\subsection{Ischemic Events and Morbidity}

Ischemic events were encountered in both groups; the rate of asymptomatic ones is higher in coiling group (21.4\%) than in clipping group (6.3\%) In contrast, the occurrence rate of symptomatic ischemic complication is higher in clipping group (49\%) than in coiling group (35.7\%) but there was no statistical significance of either of them.

After clipping there was 8 cases with early infarction (17\%) and 21 cases with late infarction (44.6\%) while in the coiling group 3 cases with early infarction (21.4\%) and 5 cases with late infarction (35.7\%). Procedure related ischemic events showed no statistical difference $(\mathrm{P}=0.7)$

The occurrence rate of temporary morbidity including limb paralysis, dysphasia, cognitive dysfunction, cranial nerve affection, CSF leak, urinary dysfunction and frontal lobe symptoms was higher in the surgery group. The other independent complications and occurrence rate in each group were also shown in Table 2.

Table 3. Clinical outcome in both groups of ruptured aneurysms.

\begin{tabular}{cccc}
\hline \multirow{2}{*}{ WFNS scale } & \multicolumn{2}{c}{ Outcome } & \multirow{2}{*}{ P-value } \\
\cline { 2 - 3 } & mRS 0 - 2 & mRS 3 - 6 & \\
\hline WFNS grade 1 - & & & 0.65 \\
Clipping (29) & $21(72.4 \%)$ & $8(27.6 \%)$ & \\
Coiling (7) & $6(85.7)$ & $1(14.3)$ & 0.06 \\
WFNS grade 4 - 5 & & & \\
Clipping (18) & $3(16.7 \%)$ & $15(83.3 \%)$ & \\
Coiling (7) & $4(57.1 \%)$ & $3(42.9 \%)$ & \\
\hline
\end{tabular}

WFNS: World Federation of Neurosurgical Societies; mRS: modified Rankin Scale.

Table 4. Mortality cases in ruptured aneurysms.

\begin{tabular}{|c|c|c|c|c|c|c|c|c|c|}
\hline & Sex & Age & Location & Dome Size & Neck Size & WFNS & Fisher & Modality & condition \\
\hline 1 & $\mathrm{M}$ & 73 & Lt. IC-AchA & 16.4 & 9.68 & 5 & 4 & Clipping & $\begin{array}{l}\text { Massive infarction with edema and } \\
\text { midline shift due to vasospasm }\end{array}$ \\
\hline 2 & $\mathrm{~F}$ & 44 & Lt. ICA & 12.47 & 3.8 & 5 & 4 & Coiling & $\begin{array}{l}\text { Massive infarction with edema and } \\
\text { midline shift due to vasospasm }\end{array}$ \\
\hline 3 & $\mathrm{~F}$ & 85 & Lt. ICPC & 11.3 & 4.2 & 5 & 3 & Coiling & $\begin{array}{l}\text { Failure of coiling, rebleeding, } \\
\text { multiple strokes and hydrocephalus }\end{array}$ \\
\hline
\end{tabular}

Lt: left, Rt: right, ICA: internal carotid artery; AchA: anterior choroidal artery; PC: posterior communicating; F: female; M: male; WFNS: World Federation of Neurosurgical Societies. 
Postoperative second intervention for complications was statistically higher in the surgery group as 17 cases underwent second surgeries, in some cases more than one surgery with total of 29 surgeries (61.7\%) while in the coiling group, 4 cases subjected to second surgeries $(28.6 \%)(\mathrm{P}=0.036)$, nature of the second intervention and its frequency is shown in Table 5. postoperative hospital period was significantly longer in clipping 44 days \pm 25 than in coiling 31.4 days \pm 18 ( $\mathrm{P}$ $=0.04)($ Table 2).

\subsection{Predictors for Neck Remnant}

Univariate analysis of risk factors for neck remnant in the clipping group presented neck width $(P=0.03)$ and the presence of incorporate artery (IA) $(P=$ 0.008 ) to be significant independent variables. The presence of IA is significant in multivariate analysis (Table 6). Regarding coiling group, analysis of neck remnant and regrowth was not possible due to small number.

\subsection{Predictors for Ischemic Events and Functional Outcome}

On analysis of cases with ischemic events (symptomatic and asymptomatic) in the surgery group, age of the patient $(\mathrm{P}=0.038)$, size of the aneurysm dome $(\mathrm{P}=$ $0.008)$, neck width $(\mathrm{P}=0.005)$, WFNS grade $4-5(\mathrm{P}=0.0001)$ and SAH with modified Fisher grade 4 in preoperative imaging $(\mathrm{P}=0.0004)$ were found to be

Table 5. Operations for complications in both groups of ruptured aneurysms.

\begin{tabular}{ccc}
\hline Modality & Intervention & Frequency \\
\hline Surgical clipping & Ventriculoperitoneal shunt & 13 \\
Cranioplasty & 3 \\
Decompressive craniectomy & 2 \\
Wound debridement & 1 \\
Evacuation of EDH & 1 \\
Dural repair for CSF leak & 1 \\
Endovascular coiling & Trapping of Acom A. (post-operative pseudoaneurysm) & 1 \\
& Ophthalmological surgery for Terson syndrome & 3
\end{tabular}

EDH: extradural hematoma; CSF: cerebrospinal fluid; Acom A.: anterior communicating artery.

Table 6. Predictors of neck remnant after clipping in ruptured aneurysms.

\begin{tabular}{|c|c|c|c|c|}
\hline \multirow{2}{*}{ Variable } & \multirow{2}{*}{$\begin{array}{c}\text { Univariate analysis } \\
\text { P value }\end{array}$} & \multicolumn{3}{|c|}{ Multivariate analysis } \\
\hline & & $\mathrm{P}$ value & Odd ratio & Confidence interval 95\% \\
\hline Neck width & & & & \\
\hline continuous & 0.14 & 0.18 & 1.6 & $0.85-3.49$ \\
\hline Categorical $(>4 \mathrm{~mm})$ & $0.03^{*}$ & & & \\
\hline Presence of IA & $0.008^{*}$ & $0.04^{*}$ & 8.5 & $1.13-80.9$ \\
\hline
\end{tabular}

*statistically significant. 
statistically significant in univariate analysis. On multivariate analysis, WFNS grade 4 - 5 was significant $(\mathrm{P}=0.01$ ) (Table 7$)$. In the coiling group analysis did not reveal significant predictors, yet it showed higher percentage of ischemic infarct with modified fisher grade 4 .

Analysis of the predictors of poor functional outcome (mRS $>2$ ) of all ruptured aneurysms reveled age $(\mathrm{P}=0.0006)$, dome size $>10 \mathrm{~mm}(\mathrm{P}=0.03)$, WFNS grade 4 - $5(\mathrm{P}=0.0002)$, modified Fisher grade $4(\mathrm{P}=0.007)$, occurrence of peri-operative ischemic events $(\mathrm{P}=0.00003)$ and occurrence of hydrocephalus $(\mathrm{P}=$ $0.0004)$ to be important risk factors for poor outcome with no effect of the modality of treatment itself. On multivariate analysis age $(\mathrm{P}=0.04)$, WFNS grade 4 $5(\mathrm{P}=0.01)$, ischemic events $(\mathrm{P}=0.04)$ and hydrocephalus $(\mathrm{P}=0.02)$ were all significant (Table 8).

\section{Discussion}

The current study was conducted in one institute but in which both treatments

Table 7. Predictors of ischemic events after clipping in ruptured aneurysms.

\begin{tabular}{ccccc}
\hline \multirow{2}{*}{ Variable } & Univariate analysis & \multicolumn{3}{c}{ Multivariate analysis } \\
\cline { 2 - 5 } & P value & P value & Odd ratio & Confidence interval 95\% \\
\hline Age & $0.038^{*}$ & 0.2 & 1.04 & $0.98-1.12$ \\
Neck width & & & & $0.32-7.65$ \\
continuous & $0.01^{*}$ & 0.65 & 1.4 & \\
Categorical (>4 mm) & $0.005^{*}$ & & & $0.67-4.85$ \\
Size of dome & & & & \\
continuous & $0.008^{*}$ & 0.28 & 1.68 & $1.9-130$ \\
Categorical (>10 mm) & 0.0 .49 & & & $0.63-16.3$ \\
WFNS grade 4 - 5 & $0.0001^{*}$ & $0.01^{*}$ & 124 & \\
Modified Fisher grade 4 & $0.0004^{*}$ & 0.27 & 2.35 & \\
\hline
\end{tabular}

WFNS: World Federation of Neurosurgical Societies; ${ }^{*}$ statistically significant.

Table 8. Predictors of poor functional outcome in ruptured aneurysms.

\begin{tabular}{ccccc}
\hline \multirow{2}{*}{ Variable } & Univariate analysis & \multicolumn{3}{c}{ Multivariate analysis } \\
\cline { 2 - 5 } & P value & P value & Odd ratio & Confidence interval 95\% \\
\hline Age & $0.0006^{*}$ & $0.04^{*}$ & 1.07 & $1.007-1.15$ \\
$\begin{array}{c}\text { Size of dome } \\
\text { continuous }\end{array}$ & 0.15 & 0.78 & 0.94 & $0.64-1.35$ \\
Categorical $(>10 \mathrm{~mm})$ & $0.03^{*}$ & & & $1.86-79.3$ \\
WFNS grade 4 - 5 & $0.0002^{*}$ & $0.01^{*}$ & 10.2 & $0.08-4.51$ \\
Modified Fisher grade 4 & $0.007^{*}$ & 0.7 & 0.69 & $1.11-37.3$ \\
Ischemic events & $0.00003^{*}$ & $0.04^{*}$ & 5.81 & $1.39-62.7$ \\
hydrocephalus & $0.0004^{*}$ & $0.02^{*}$ & 7.95 &
\end{tabular}

WFNS: World Federation of Neurosurgical Societies; *statistically significant. 
have been always available, we demonstrated that ruptured aneurysms located in anterior circulation were possible to be treated safely by clipping or coiling. The durability was greater in clipping group than in coiling group. Although the functional outcome did not show statistical difference between the two modalities, coiling was associated with better outcome than clipping especially in severe cases with preoperative WFNS 4 - 5 and shorter postoperative hospital period.

\subsection{Durability}

Regarding efficacy of treatment, previous reports show superiority of clipping over coiling in ruptured aneurysms in aneurysm obliteration, presence of residual aneurysm, recurrence and retreatment. In Barrow Ruptured Aneurysm Trial (BRAT) study, They achieved complete obstruction of aneurysms after 6 years follow up in $96 \%$ after clipping and $48 \%$ after coiling $(\mathrm{P}<0.0001)$ with $4.6 \%$ retreatment rate in the clipping group and $16.4 \%$ retreatment rate in the coiling group $(\mathrm{P}<0.0001)$ [7]. H. Li et al. conducted a metanalysis for studies comparing clipping and coiling in ruptured aneurysm, in this analysis they included Five studies [8] [9] [10] [11] [12] with 1749 patients, the results of the analysis supported the superiority of clipping over coiling regarding complete aneurysm occlusion (84.0\% versus $66.5 \%)$ [13].

Our results have similar trends to the previous studies showing high occlusion rate in clipping over coiling. Post-operative neck remnant after clipping occurred in 9 cases (19.2\%) in our study, Intentional incomplete clipping is necessary with a certain probability to avoid the complications. We demonstrated that aneurysms with neck width $>4 \mathrm{~mm}$ and presence of incorporate artery are risk factor of incomplete clipping which is in line with previous reports [14]. The significant predictor factor in multivariate analysis in the current study is the presence of incorporate artery, which is explainable as in attempt to keep the incorporate artery patent, part of the neck is not clipped, Ahn and Kim did not show the statistical significance but they pointed out that it is one of the unavoidable causes of neck remnant [15].

Size of the aneurysmal sac is a strong predictor for neck remnant after clipping in previous studies [14], we did not find this association in the current study because most of the ruptured aneurysm in this study are small in size with 95.7\% of ruptured cases are less than $10 \mathrm{~mm}$.

Regrowth and retreatment after coiling is higher than after clipping [7] [13]. Gallas et al. demonstrated regrowth rate $14.8 \%$ retreatment $4.7 \%$ after coiling in her study [3], while recurrent aneurysms needed retreatment was $11.9 \%$ in Sluzewski et al. [16]. In our study 2 cases (14.2\%) showed coil compaction, one after 4 months and the second after 16 months. both cases showed residual dome filling immediately after coiling. they are under follow up with no retreatment required yet.

Recurrence and recanalization after coiling usually results from coil compaction either due to increased blood flow at the aneurysm site or continued growth 
of the aneurysm due to vessel disease [3]. In many reports coiling of ruptured aneurysm yields better functional outcome, nonetheless the risk of recurrence, retreatment and rebleeding is higher after coil embolization [17], even the results of the Cerebral Aneurysm Rerupture After Treatment (CARAT) study, Which demonstrated similar results between clipping and coiling in regard to rehemorrhage rates, it indicated the presence of strong association between rebleeding risk and the size of residual aneurysm [7].

O'Kelly et al. in his administrative analysis suggested that hemorrhage free survival is worse after coiling of ruptured aneurysms and that the ISAT results although it shows superiority of coiling over clipping regarding mortality after 1 year follow up, yet in the patients with longer follow up (mean 9 years) mortality became similar between the two groups, this indicates that initial better outcome of coiling is diminished by time as a result of rehaemorrhage caused by increased recurrence rate [18], which makes durability more and more important aspect due to its effect on the long-term functional outcome.

In the light of these results, it is recommended to use the maximum possible number of platinum coils in the treatment of aneurysms to achieve good aneurysm packing and avoid recurrence and reheomrrhage. It is demonstrated by several histologic studies that coil compaction is increased when the initial packing attenuation is less than $20 \%$ and coil packing attenuation more than $25 \%$ is protective against recanalization [3] [19]. Regarding durability, Ruptured aneurysms in very young patients especially those who are difficult to perform regular follow up are better treated by clipping than coiling to avoid the risk of rebleeding and retreatment on the long run.

\subsection{Ischemic Events}

In our study, ischemic events occurred in 26 cases (55.3\%) after clipping ( 3 silent + 23 symptomatic) and in 8 cases $(57.1 \%)$ after coiling ( 3 silent +5 symptomatic) which is almost similar $(\mathrm{P}=0.38)$, also procedure related ischemic events showed no statistical difference between clipping and coiling (17\% versus $21.4 \%$, $\mathrm{P}=0.7)$ which is in line with previous reports [13] [20].

The incidence of ischemic infarcts after SAH varies in comparative studies of clipping and coiling for ruptured aneurysm occlusion [13]. In the current study vasospasm related infarction was $44.6 \%$ and $35.7 \%$ in clipping and coiling respectively with no statistical difference between the two modalities $(\mathrm{P}=0.5)$.

Although many studies found total vasospasm (asymptomatic \& symptomatic) to be more after clipping, most of them reported no difference between the two modalities of treatment regarding the actual occurrence of cerebral infarction which validate our results.

It is a matter of speculation as to why the type of treatment of the ruptured aneurysm might affect vasospasm. There are arguments in favor of clipping to decrease vasospasm as a result to removal of cisternal blood during surgery, an argument against this is the lack of removal of cisternal blood and clots during 
coiling yet it shows lower rates of vasospasm in previous reports [13] [21], however other factors may be involved with increased vasospasm after clipping as craniotomy, brain retraction and manipulation of the blood vessels during surgery [22]. Previous studies reported an association between vasospasm and cerebral infarction [21] [22]. Therefore, an increase in angiographic vasospasm attributable to surgical clipping also should be associated with increased cerebral infarction. This was not the case in this analysis as our results and previous reports show no difference between clipping and coiling in that matter.

The current study presented the size of aneurysm dome and neck, age, WFNS grade 4 - 5 and Modified fisher Grade 4 as independent factors for ischemic events after clipping. This is in line with other reports [22] [23]. In the multivariate analysis WFNS grade 4 - 5 was significant and this actually explains the high incidence of ischemic events in the current study as $41 \%$ of the patients had poor preoperative grade (WFNS grade 4 - 5).

\subsection{Functional Outcomes and Morbidity}

Since the publication of the results of the ISAT study in 2002 and the management of ruptured intracranial aneurysms changed dramatically in favor of endovascular coiling. Along with this change, the debate increased in the scientific community regarding clipping and coiling and which modality is more superior regarding functional outcomes. The ISAT study at one year follow up showed Good outcome (mRS $0-2$ ) $74.6 \%$ and $63.6 \%$ in coiling and clipping respectively [24]. But the ISAT study population showed $88 \%$ of patients with WFNS grade 1 - 2, also as we mentioned before at 6 years follow up the mortality between the two groups did not show much difference due to increased rate of rebleeding and retreatment in the coiling group [18].

Three years after the ISAT, Kato et al. demonstrated that clipping is more superior to coiling with $64.4 \%$ good outcome after clipping and $38.2 \%$ good outcome after coiling, with so much criticism to the ISAT study results [25]. In 2013, H. Li et al. conducted a meta-analysis for studies comparing clipping and coiling in ruptured aneurysms, in this meta-analysis RCTs demonstrated better outcomes with coiling than clipping $(\mathrm{P}<0.00001)$, while the observational studies showed no difference between the two modalities in outcomes $(\mathrm{P}=0.17)$. In patients with poor preoperative grade (WFNS/Hunt \& Hess grade 4 - 5), there was no significant difference in outcome between clipping and coiling. Both observational studies and RCTs found no statistical difference between the two modalities regarding mortality [13].

In 2015, the 6-year results of the Barrow Ruptured Aneurysm Trial (BRAT) was published, they noted that there was no significant difference in the functional outcome between the two modalities in the treatment of ruptured anterior circulation aneurysms at almost all time points in BRAT. The results of the 6 year follow up showed $34 \%$ and $39 \%$ poor outcome in the coil-assigned group and clip-assigned group respectively $(\mathrm{P}=0.33)$ [7]. 
The current study shows poor outcome in 23 patients (49\%) after clipping and 4 patients (28.6\%) after coiling with no statistical difference between the two groups $(\mathrm{P}=0.22)$. Although statistically insignificant our results showed a better percent of good outcome in the coiling group especially in cases with WFNS grade $4-5$. However, whether these results and the difference between the two modalities will hold and exist on the long run or not is a matter of debate, as there is a trend in the reports with long follow up for the results of functional outcome to become more similar with time due to the effect of durability of each modality. Another aspect in our results that poor outcome for both modalities is higher than in previous reports, this is because patients with WFNS grade 4 - 5 constituted $<5 \%$ and $16 \%$ in ISAT and BRAT respectively [7], while in our study they constitute $41 \%$.

Our data presented age, size of the aneurysm dome, WFNS grade 4 - 5, Modified Fisher grade 4, Ischemic events and hydrocephalus as important predictors for poor outcome in univariate analysis. In multivariate analysis age, WFNS grade $4-5$, ischemic events and hydrocephalus were significant. The type of treatment modality did not significantly affect outcome in this study $(P=0.2)$. these results are consistent with previous reports [16] [18] [20] [23].

The PRESAT group found many significant predictors for poor outcomes, On multivariate analyses, poor outcomes were associated with increasing age, WFNS grade IV-V on admission, preadmission aneurysmal rerupture, vasospasm-induced cerebral infarct, infection (pneumonia and sepsis), shunt-dependent hydrocephalus, seizure, postclipping hemorrhagic complications, and postcoiling ischemic complications [20], but the type of treatment modality was not significant in either univariate or multivariate analyses which support our results.

Hydrocephalus is a well-known sequelae of aneurismal SAH and an important predictor for poor outcome in this study, has been reported to range from $6 \%$ to 67\% [9]. It occurred in 13 cases (27.6\%) after clipping and 4 cases (28.6\%) after coiling in the current study $(\mathrm{P}=1)$. There is an argument that clipping decreases the frequency of hydrocephalus by removing blood clots from the subarachnoid space and lamina terminalis fenestration [25], that was not the case in our study with no effect of the modality of treatment on the frequency of hydrocephalus, our results are supported by previous results as Seven studies [9] [26]-[31] (1981 patients) demonstrated no difference between clipping and coiling regarding shunt depending hydrocephalus $(16.4 \%$ versus $19.3 \%$; $=0.16)$ [13].

Hemorrhagic complications in the current study occurred mainly with clipping as a result of craniotomy, most of them were asymptomatic and did not require treatment and did not affect the patient outcome, only one case of extradural hematoma needed surgical intervention. The rest of surgical interventions required after craniotomy were mainly for hydrocephalus (which had similar rate between clipping and coiling), followed by decompressive craniotomy and cranioplasty which were done mainly in 5 out of 7 cases in patients with WFNS grade $4-5$, in 4 cases of them the decision to perform decompressive 
craniectomy was taken during the aneurysm surgery due to brain swelling inevitably leading to a second intervention for cranioplasty.

Our data showed that clipping as compared to coiling was associated with an average of 1.4-times more days in hospitalization for ruptured patients. Hoh et al. demonstrated similar trend regarding hospitalization period [32], we believe the invasiveness of the procedure and the need for second intervention played a role in this difference.

\subsection{Mortality}

Three patients died during this study, one case after clipping and two after coiling $(\mathrm{P}=0.2)$ with no difference between the two modalities in that matter and this is consistent with previous reports [7] [13] [17]. All the patients were WFNS grade 5 and died from cerebral infarctions related to vasospasm. Mortality in this study was evaluated at $4.9 \%$, because of poor preoperative conditions. High mortality rate after severe SAH is expected, Bracard et al. reported results involving severe hemorrhage, with a global mortality rate of $29 \%$ [33]. The actual rates of morbidity and mortality of clipping and coiling in ruptured aneurysms are difficult to be evaluated as a result of SAH and its complications, they are best evaluated in unruptured aneurysms [3].

\section{Conclusion}

In the treatment of ruptured aneurysms, both clipping and coiling are feasible options. Clipping has the advantage of durability and less recurrence which makes it more suitable for very young patients or patients whose commitment to follow up is not certain. coiling is associated with better functional outcome especially in the more severe cases, so whenever it is available it should be used for the treatment but with precautions, good coil packing is essential to avoid recurrence of the aneurysm and good follow up to detect the recurrence when it happens to avoid rebleeding complications. Nowadays, for anterior circulation aneurysm, if both treatments are feasible, coil embolization may be preferred, which is the current point proved by the RCT like ISAT. But if you choose the patients carefully and find out the optimal candidate for the clipping, the clipping is still enough feasible as a mainstream.

\section{Limitations}

This study has some limitations, first it is a retrospective study reviewed from only medical record. Surgical indication and treating method were determined by neurosurgeon individually. Secondary, it has small number of patients especially for the endovascular group. Third, the follow up period was short to evaluate the regrowth and rebleeding in the future.

\section{Conflicts of Interest}

The authors declare no conflicts of interest regarding the publication of this paper. 


\section{References}

[1] Zacharia, B.E., et al. (2010) Epidemiology of Aneurysmal Subarachnoid Hemorrhage. Neurosurgery Clinics of North America, 21, 221-233. https://doi.org/10.1016/j.nec.2009.10.002

[2] Rabinstein, A.A. (2013) About Subarachnoid Hemorrhage. Neurology, 80, e56-e59. https://doi.org/10.1212/WNL.0b013e3182834b22

[3] Gallas, S., et al. (2005) A Multicenter Study of 705 Ruptured Intracranial Aneurysms Treated with Guglielmi Detachable Coils. American Journal of Neuroradiology, 26, 1723-1731.

[4] Moret, J., Cognard, C., Weill, A., Castaings, L. and Rey, A. (1997) The "Remodelling Technique" in the Treatment of Wide Neck Intracranial Aneurysms. Interventional Neuroradiology, 3, 21-35. https://doi.org/10.1177/159101999700300103

[5] Shapiro, M., Babb, J., Becske, T. and Nelson, P.K. (2008) Safety and Efficacy of Adjunctive Balloon Remodeling during Endovascular Treatment of Intracranial Aneurysms: A Literature Review. American Journal of Neuroradiology, 29, 1777-1781. https://doi.org/10.3174/ajnr.A1216

[6] Connolly, E.S., et al. (2012) AHA/ASA Guideline Guidelines for the Management of Aneurysmal Subarachnoid Hemorrhage: A Guideline for Healthcare Professionals from the American Heart Association/American Stroke Association. Stroke, 43, 1711-1737. https://doi.org/10.1161/STR.0b013e3182587839

[7] Spetzler, R.F., et al. (2015) The Barrow Ruptured Aneurysm Trial: 6-Year Results. Journal of Neurosurgery, 123, 609-617. https://doi.org/10.3171/2014.9.JNS141749

[8] Molyneux, A.J., et al. (2005) International Subarachnoid Aneurysm Trial (ISAT) of Neurosurgical Clipping versus Endovascular Coiling in 2143 Patients with Ruptured Intracranial Aneurysms: A Randomised Comparison of Effects on Survival, Dependency, Seizures, Rebleeding, Subgroups, and Aneurysm Occlusion. The Lancet (London, England), 366, 809-817. https://doi.org/10.1016/S0140-6736(05)67214-5

[9] Taha, M.M., et al. (2006) Endovascular Embolization vs Surgical Clipping in Treatment of Cerebral Aneurysms: Morbidity and Mortality with Short-Term Outcome. Surgical Neurology, 66, 277-284. https://doi.org/10.1016/j.surneu.2005.12.031

[10] Koivisto, T., Vanninen, R., Hurskainen, H., Saari, T., Hernesniemi, J. and Vapalahti, M. (2000) Outcomes of Early Endovascular versus Surgical Treatment of Ruptured Cerebral Aneurysms. A Prospective Randomized Study. Stroke, 31, 2369-2377. https://doi.org/10.1161/01.STR.31.10.2369

[11] Proust, F., et al. (2003) Treatment of Anterior Communicating Artery Aneurysms: Complementary Aspects of Microsurgical and Endovascular Procedures. Journal of Neurosurgery, 99, 3-14. https://doi.org/10.3171/jns.2003.99.1.0003

[12] de los Reyes, K., Patel, A., Bederson, J.B. and Frontera, J.A. (2013) Management of Subarachnoid Hemorrhage with Intracerebral Hematoma: Clipping and Clot Evacuation versus Coil Embolization Followed by Clot Evacuation. Journal of NeuroInterventional Surgery, 5, 99-103. https://doi.org/10.1136/neurintsurg-2011-010204

[13] Li, H., et al. (2013) Clipping versus Coiling for Ruptured Intracranial Aneurysms: A Systematic Review and Meta-Analysis. Stroke, 44, 29-37. https://doi.org/10.1161/STROKEAHA.112.663559

[14] Jabbarli, R., et al. (2016) Aneurysm Remnant after Clipping: The Risks and Consequences. Journal of Neurosurgery, 125, 1249-1255. https://doi.org/10.3171/2015.10.JNS151536 
[15] Ahn, S.S. and Kim, Y.D. (2010) Three-Dimensional Digital Subtraction Angiographic Evaluation of Aneurysm Remnants after Clip Placement. Journal of Korean Neurosurgical Society, 47, 185-190. https://doi.org/10.3340/jkns.2010.47.3.185

[16] Sluzewski, M., Van Rooij, W.J., Rinkel, G.J.E. and Wijnalda, D. (2003) Endovascular Treatment of Ruptured Intracranial Aneurysms with Detachable Coils: Long-Term Clinical and Serial Angiographic Results. Radiology, 227, 720-724. https://doi.org/10.1148/radiol.2273020656

[17] Lanzino, G., Murad, M.H., D’Urso, P.I. and Rabinstein, A.A. (2013) Coil Embolization versus Clipping for Ruptured Intracranial Aneurysms: A Meta-Analysis of Prospective Controlled Published Studies. American Journal of Neuroradiology, 34, 1764-1768. https://doi.org/10.3174/ajnr.A3515

[18] O’Kelly, C.J., Kulkarni, A.V., Austin, P.C., Wallace, C. and Urbach, D. (2010) The Impact of Therapeutic Modality on Outcomes Following Repair of Ruptured Intracranial Aneurysms: An Administrative Data Analysis. Journal of Neurosurgery, 113, 795-801. https://doi.org/10.3171/2009.9.JNS081645

[19] Taussky, P., Kallmes, D.F. and Cloft, H. (2012) Mathematic Analysis of Incremental Packing Density with Detachable Coils: Does That Last Coil Matter Much? American Journal of Neuroradiology, 33, 74-75. https://doi.org/10.3174/ajnr.A2454

[20] Taki, W. (2011) Determinants of Poor Outcome after Aneurysmal Subarachnoid Hemorrhage When Both Clipping and Coiling Are Available: Prospective Registry of Subarachnoid Aneurysms Treatment (PRESAT) in Japan. World Neurosurgery, 76, 437-445. https://doi.org/10.1016/j.wneu.2011.04.026

[21] Hohlrieder, M., et al. (2002) Cerebral Vasospasm and Ischaemic Infarction in Clipped and Coiled Intracranial Aneurysm Patients. European Journal of Neurology, 9, 389-399. https://doi.org/10.1046/j.1468-1331.2002.00425.x

[22] Dumont, A.S., et al. (2010) Endovascular Treatment or Neurosurgical Clipping of Ruptured Intracranial Aneurysms: Effect on Angiographic Vasospasm, Delayed Ischemic Neurological Deficit, Cerebral Infarction, and Clinical Outcome. Stroke, 41, 2519-2524. https://doi.org/10.1161/STROKEAHA.110.579383

[23] Hoh, B.L., et al. (2004) Effect of Clipping, Craniotomy, or Intravascular Coiling on Cerebral Vasospasm and Patient Outcome after Aneurysmal Subarachnoid Hemorrhage. Neurosurgery, 55, 779-789. https://doi.org/10.1227/01.NEU.0000137628.51839.D5

[24] Molyneux, A. and Kerr, R. (2002) International Subarachnoid Aneurysm Trial (ISAT) of Neurosurgical Clipping Versus Endovascular Coiling in 2143 Patients with Ruptured Intracranial Aneurysms: A Randomized Trial. Journal of Stroke and Cerebrovascular Diseases, 11, 304-314. https://doi.org/10.1053/jscd.2002.130390

[25] Kato, Y., et al. (2005) The Effect of Clipping and Coiling in Acute Severe Subarachnoid Hemorrhage after International Subarachnoid Aneurysmal Trial (ISAT) Results. Minimally Invasive Neurosurgery, 48, 224-227. https://doi.org/10.1055/s-2005-870930

[26] Dehdashti, A.R., Rilliet, B., Rufenacht, D.A. and de Tribolet, N. (2004) Shunt-Dependent Hydrocephalus after Rupture of Intracranial Aneurysms: A Prospective Study of the Influence of Treatment Modality. Journal of Neurosurgery, 101, 402-407. https://doi.org/10.3171/jns.2004.101.3.0402

[27] Gruber, A., Reinprecht, A., Bavinzski, G., Czech, T. and Richling, B. (1999) Chronic Shunt-Dependent Hydrocephalus after Early Surgical and Early Endovascular Treatment of Ruptured Intracranial Aneurysms. Neurosurgery, 44, 503-509. https://doi.org/10.1097/00006123-199903000-00039 
[28] Natarajan, S.K., Sekhar, L.N., Ghodke, B., Britz, G.W., Bhagawati, D. and Temkin, N. (2008) Outcomes of Ruptured Intracranial Aneurysms Treated by Microsurgical Clipping and Endovascular Coiling in a High-Volume Center. American Journal of Neuroradiology, 29, 753-759. https://doi.org/10.3174/ajnr.A0895

[29] de Oliveira, J.G., et al. (2007) Risk of Shunt-Dependent Hydrocephalus after Occlusion of Ruptured Intracranial Aneurysms by Surgical Clipping or Endovascular Coiling: A Single-Institution Series and Meta-Analysis. Neurosurgery, 61, 924-933.

[30] Varelas, P., Helms, A., Sinson, G., Spanaki, M. and Hacein-Bey, L. (2006) Clipping or Coiling of Ruptured Cerebral Aneurysms and Shunt-Dependent Hydrocephalus. Neurocritical Care, 4, 223-228. https://doi.org/10.1385/NCC:4:3:223

[31] Nam, K.-H., Hamm, I.-S., Kang, D.-H., Park, J. and Kim, Y.-S. (2010) Risk of Shunt Dependent Hydrocephalus after Treatment of Ruptured Intracranial Aneurysms: Surgical Clipping versus Endovascular Coiling According to Fisher Grading System. Journal of Korean Neurosurgical Society, 48, 313-318. https://doi.org/10.3340/jkns.2010.48.4.313

[32] Hoh, B.L., Chi, Y.Y., Lawson, M.F., Mocco, J. and Barker, F.G. (2010) Length of Stay and Total Hospital Charges of Clipping versus Coiling for Ruptured and Unruptured Adult Cerebral Aneurysms in the Nationwide Inpatient Sample Database 2002 to 2006. Stroke, 41, 337-342. https://doi.org/10.1161/STROKEAHA.109.569269

[33] Bracard, S., et al. (2002) Endovascular Treatment of Hunt and Hess Grade IV and V Aneuryms. American Journal of Neuroradiology, 23, 953-957. 


\section{Abbreviations}

ACA, Anterior Cerebral Artery

AHA/ASA, American Heart Association/American Stroke Association

BRAT, Barrow Ruptured Aneurysm Trial

CARAT, The Cerebral Aneurysm Rerupture after Treatment Study

CT, Computed Tomography

CTA, Computed Tomography Angiography

CSF, Cerebrospinal Fluid

DSA, Digital Subtraction Angiography

EVT, Endovascular Treatment

IA, Incorporate Artery

ICA, Internal Carotid Artery

ISAT, International Subarachnoid Aneurysm Trial

MCA, Middle Cerebral Artery

MRA, Magnetic Resonance Angiography

MRI, Magnetic Resonance Imaging

mRS, Modified Rankin Scale

PRESAT, The Prospective Registry of Subarachnoid Aneurysms Treatment Study RCT, Randomized Controlled Trials

SAH, Subarachnoid Hemorrhage

SD, Standard Deviation

WFNS, World Federation of Neurological Surgeons 